\title{
INNOVACIÓN Y VIRTUALIZACIÓN PROGRESIVAS DE LAS UNIVERSIDADES IBEROAMERICANAS HACIA LA SOCIEDAD DEL CONOCIMIENTO
}

\author{
(PROGRESSIVE VIRTUALIZATION AND INNOVATION OF THE IBEROAMERICAN UNIVERSITIES \\ IN THE KNOLWLEDGE SOCIETY)
}

\author{
Miguel Casas Armengol \\ Universidad Nacional Abierta, Universidad del Zulia (Venezuela) \\ Lily Stojanovic \\ Universidad Central de Venezuela/ Universidad Nacional Abierta (Venezuela)
}

\section{RESUMEN}

En cualquier sociedad, sea ella "desarrollada" o "en desarrollo", el factor clave para su progreso es su efectiva capacidad para generar, y aplicar continuamente el "Conocimiento" a los diversos campos de la vida social, técnica y científica. En consecuencia, el instrumento principal y esencial para los complejos procesos de transformación y modernización de estas sociedades es la educación y dentro de ella especialmente la universitaria. Pero no se trata de la educación y la universidad en sus formas usuales y tradicionales, sino de todo un repensar en formas nuevas de organización, administración, investigación y acción. Estos cambios pueden impulsarse mediante teorías y procesos innovadores que permiten las nuevas tecnologías de la información y comunicación.

En este trabajo se examina esta transformación de universidades en base a la tríada de la innovación, virtualización y globalización.

\begin{abstract}
In any society developed or underdeveloped the key factor for its progress would be its possibility for generating and applying knowledge in the social, technical and cientific areas. For attaining the goal of the complex process of transformation and modernization of such societies the main instrument would be education, specially at the university level, but redesigned in new ways. This paper examines how progressive virtualization and innovation of higher education institutions could play a significant role in that transformation.
\end{abstract}




\section{INTRODUCCIÓN}

Casas (2005) plantea que en cualquier sociedad Iberoamericana, sea ella "desarrollada" o "en desarrollo", el factor clave para su progreso es su efectiva capacidad para generar, y aplicar continuamente el "Conocimiento" a los diversos campos de la vida social, técnica y científica. En consecuencia, el instrumento principal y esencial para los complejos procesos de transformación y modernización de estas sociedades es la educación y dentro de ella especialmente la universidad. Pero no se trata de la educación y la universidad en sus formas usuales y tradicionales, sino de todo un repensar de formas nuevas y diferentes, tanto de la educación como de la institución universitaria, impulsando tales cambios mediante los nuevos procesos y teorías de la moderna innovación. En apoyo de estos supuestos, Castells (2001) concluye que estos configuran una nueva sociedad que él denomina como la "Sociedad Red" y expresa:

"Nuestra exploración de las estructuras sociales emergentes por distintos ámbitos de la actividad y experiencia humana conducen a una conclusión general: como tendencia histórica, las funciones y los procesos dominantes en la era de la información cada vez se organizan más en torno a "redes". Estas constituyen la nueva morfología social de nuestras sociedades y la difusión de su lógica de enlace modifica de forma sustancial la operación y los resultados de los procesos de producción, la experiencia, el poder y la cultura”.

También Brünner (2003) señala los grandes cambios de la sociedad latinoamericana actual cuando examina la denominada revolución digital, impulsada por dos grandes fuerzas estrechamente imbricadas: la globalización por un lado y los efectos causados por las nuevas tecnologías de la información (TIC).

\section{Universidad y la Sociedad del Conocimiento}

En un mundo como el actual, caracterizado por un cambio incesante e inesperado, y por una creciente Globalización, el paradigma clásico de una universidad tradicional y casi inmutable, no resulta muy congruente con las nuevas realidades y demandas sociales y científicas, tanto actuales como futuras. Por otra parte, si consideramos que, cada vez más, importantes investigaciones coinciden en afirmar que ninguna sociedad actual es superior a sus universidades, resulta evidente que un instrumento esencial del progreso y el desarrollo es la universidad. En efecto, no 
Miguel CASAS y LiLy STOJANOVIC

INNOVACIÓN Y VIRTUALIZACIÓN PROGRESIVAS DE LAS UNIVERSIDADES IBEROAMERICANAS HACIA LA SOCIEDAD DEL CONOCIMIENTO

hay países realmente avanzados que no cuenten con un eficaz sistema universitario $\mathrm{y}$, dentro de él, con unas sólidas y permanentes investigaciones. Estas afirmaciones revisten especial importancia para el caso de Iberoamérica, al constatar que aún algunas de sus universidades más destacadas, también están evidenciando, hoy día, serias y continuas limitaciones para poder modificar rápida y profundamente sus modelos, estructuras y procedimientos obsoletos, a fin de poder responder funcional y oportunamente, a las nuevas y exigentes demandas.

Los sistemas de educación superior, dice la Declaración Mundial de la UNESCO (París, octubre de 1998), deberían aumentar su capacidad para vivir en medio de la incertidumbre para transformarse y provocar el cambio. La incertidumbre no debe conducirnos a la perplejidad, sino a la disposición para el cambio y a la ampliación y renovación incesante del conocimiento. Si el siglo XX fue el siglo de la búsqueda de certezas científicas y del desarrollo acelerado de las diferentes disciplinas del conocimiento humano, el presente siglo está llamado a ser el siglo de la incertidumbre y la interdisciplinariedad. Por consiguiente, si la universidad es un instrumento tan decisivo para las sociedades iberoamericanas, resulta inaplazable poder transformar profundamente, tanto sus instituciones individuales como sus sistemas, redes y conjuntos universitarios; y de esta transformación dependerá que la universidad pueda impulsar que sus sociedades tradicionales o estáticas se orienten a convertirse en modernas sociedades de la información y del conocimiento.

La idea es resaltar la extraordinaria importancia de poder incorporar el instrumentoy proceso dela Innovación en su moderna acepción, para realizar cambios sustanciales, integrados y prospectivos en las universidades iberoamericanas, a fin de lograr que estas adquieran una decisiva trascendencia para la transformación y modernización de sus sociedades. Para destacar la importancia de este moderno concepto de innovación, Albornoz (2002) afirma: "La Innovación es la base de la Sociedad del Conocimiento y uno de los motores de la Globalización“ y luego agrega: "para que el desarrollo social y humano, sea sostenible, la Innovación deberá regirse por valores éticos y morales“. En realidad y tal como lo expresan diversos autores, lo que importa en lo relativo a los modernos cambios de las universidades de esta Región, es que estas se hagan mediante Innovaciones apoyadas en las modernas tecnologías que constituyen los medios (denominados ahora como de Virtualización) que permitan un nuevo contexto para las maneras de aprender, enseñar y gestionar. Pero hay que advertir que estos procesos de Innovación también comportan tensiones sociales y, por consiguiente, la dinámica de la Innovación mal utilizada, también puede contribuir a aumentar la brecha actual entre países muy avanzados y otros de muy bajo desarrollo, como es el caso, especialmente, de varias sociedades de América Latina. 
Dentro de esta problemática, el nuevo concepto de "Pertinencia" desempeña igualmente un papel clave y esencial para que las ideas, las funciones y los productos de la educación superior no se sigan limitando exageradamente a la tradicional graduación de profesionales de disciplinas liberales. A este respecto Vessuri (1998), sostiene que la "Pertinencia" es uno de los factores claves para la educación superior en el Siglo XXI y agrega:

"El término Pertinencia ha sido utilizado para referirse a la coincidencia entre lo que las instituciones de educación superior hacen y lo que la sociedad espera de ellas. Se refiere especialmente al papel y el lugar de la educación superior en la sociedad, pero también comprende el acceso y la participación, la enseñanza y el aprendizaje, la función de la universidad como centro de investigación, la responsabilidad de la educación superior con otros sectores de la sociedad, el mundo laboral y la función de servicios de educación superior en la comunidad. No menos importante es la participación de la educación superior en la búsqueda de soluciones a problemas humanos urgentes, como la población, el medio ambiente, la paz y el entendimiento internacional, así como la democracia y los derechos humanos".

\section{El conocimiento en la transformación de la universidad de la Región}

Refiriéndose al rol del conocimiento actual en la universidad Yarzábal (2000) advierte:

"el conocimiento es diferente y se gestiona de modo diferente. El conocimiento no es más monodisciplinario, es interdisciplinario, está centrado en el problema, no en la disciplina, se produce en diversos ámbitos, más cercanos a su aplicación, se ha desplazado de los ámbitos académicos para acercarse a los ámbitos productivos empresariales e industriales. Usa muy intensamente las redes electrónicas para intercambiarse, para producirse y para transformarse en tecnología, y está sujeto a controles de calidad diversificados, de tal manera, que el sólo control de la evaluación por pares ha pasado a ser obsoleto. Ahora tiene que demostrar su pertinencia social, tiene que demostrar su eficiencia económica, tiene que demostrar su calidad de otras formas, que superan a la evaluación por pares". 
Por consiguiente, de lo dicho anteriormente, resulta evidente el rol crucial que deberá jugar la universidad para lograr que las respectivas sociedades tradicionales avancen hacia la conformación, en primer lugar, de la Sociedad de la Información y en último término, idealmente, la Sociedad del Conocimiento. Para avanzar hacia tales objetivos, Tunnerman (2000) señala:

"La médula del problema radica, según lo han hecho ver numerosos autores, es que nos encontramos en una etapa de transición y por lo mismo crítica, entre la educación superior elitista y la educación superior masiva. Las actuales estructuras académicas responden a la educación superior elitista; por lo mismo, les es imposible, sin una profunda transformación, hacer frente al fenómeno de masificación. Son, en general demasiado rígidas, poco diversificadas, y carentes de adecuados canales de comunicación entre sus distintas modalidades y con el mundo de la producción y del trabajo. La homogeneidad de sus programas no les permite atender la amplia gama de habilidades, intereses y motivaciones de una población estudiantil cada vez más extensa y heterogénea; su excesiva compartimentalización contradice la naturaleza esencialmente interdisciplinaria del conocimiento moderno; su apego a los sistemas formales les impide servir con eficacia los propósitos de la educación permanente". (pp. 100-101).

Pero en el proceso de superación de las limitaciones anteriores, el conocimiento jugará continuamente un papel más preponderante, tanto en la reestructuración de la universidad como en la transformación de la sociedad y la ciencia de la región. Dada la importancia de tales supuestos, citaremos algunas precisiones de Tunnermann \& Chaui (2003):

"Una de las características de la sociedad contemporánea es el papel central del conocimiento en los procesos productivos, al punto que el calificativo más frecuente que suele dársele es el de sociedad del conocimiento. Asistimos a la emergencia de un nuevo paradigma económico-productivo en el cual el factor más importante no es ya la disponibilidad de capital, mano de obra, materias primas o energía, sino el uso intensivo del conocimiento y la información".

Hoy día, las economías más avanzadas se basan en la mayor disponibilidad de conocimiento. Las ventajas comparativas dependen cada vez más del uso competitivo 
del conocimiento y de las innovaciones tecnológicas. De allí la importancia de la Educación, pero principalmente de la Universidad, como factores fundamentales para la transformación y progreso de las sociedades actuales, sean ellas desarrolladas o subdesarrolladas. Naturalmente, en cada caso, las estrategias requeridas tendrán grandes diferencias y las de mayor complejidad y dificultad, corresponderán principalmente a aquellas sociedades que acusen un escaso desarrollo y dispongan todavía de unas instituciones y sistemas educativos, de insuficiente consolidación. Por ello, la UNESCO en su reciente declaración Mundial sobre la Educación Superior, reconoce la importancia estratégica de dicha educación en la sociedad contemporánea y concluye que: "la propia educación superior ha de emprender su transformación y la renovación más radical que jamás haya tenido por delante”.

\section{Diferenciación de paradigmas}

Un cambio trascendental en estos procesos de transformación universitaria, se refiere al cambio de paradigma de la enseñanza y el aprendizaje, en contraste con el paradigma tradicional, cuyo énfasis ha sido en la enseñanza y en el docente. Esto no significa que pierdan importancia ni la enseñanza y sus teorías, ni tampoco el profesor. De lo que se trata es que el rol del docente cambia y en vez de ser quien centra la información, él pasa a ser un guía, un tutor y un orientador, para el alumno y su aprendizaje. Las razones de estos cambios son explicadas por Tünnermann (2003):

"Los procesos actuales de transformación buscan superar la
concepción de la educación como simple "transmisión-acumulación"
de conocimientos e información. De esta manera, la llamada "crisis
educativa" es en buena parte una crisis del modelo pedagógico
tradicional. Dicho modelo requiere una revisión a fondo frente al
hecho incuestionable del crecimiento acelerado del conocimiento
contemporáneo, acompañadodesurápida obsolescencia, asícomoante
otra realidad como lo es el desplazamiento del aparato escolar como
único oferente de educación, y el surgimiento de la "escuela paralela"
de los medios masivos de comunicación y la rápida difusión de la
información a través de las modernas tecnologías. Indiscutiblemente,
estos fenómenos modifican, necesariamente, el modelo pedagógico y
el rol del profesor universitario".

Continúa el mismo autor, citando conclusiones de la Conferencia Mundial de la UNESCO (1998), en relación a que estos desafíos del aprendizaje conducen 
a respuestas académicas que forman el núcleo de los procesos actuales de transformación universitaria, y que deben inspirar los modelos educativos y académicos. Esas respuestas son:

* La adopción del paradigma del aprender a aprender.

* El traslado del acento, en la relación enseñanza-aprendizaje, a los procesos de aprendizaje.

* El nuevo rol de los docentes, ante el protagonismo de los discentes en la construcción del conocimiento significativo.

* La flexibilidad curricular y toda la moderna teoría curricular, que se está aplicando en el rediseño de los planes de estudio.

* La promoción de una mayor flexibilidad en las estructuras académicas.

* El sistema de créditos.

* La estrecha interrelación entre las funciones básicas de la universidad (docencia, investigación, extensión y servicios ).

* La redefinición de las competencias profesionales.

* La reingeniería institucional y la gestión estratégica como componente normal de la administración universitaria.

* La autonomía universitaria responsable.

* Los procesos de vinculación con la sociedad y sus diferentes sectores (productivo, laboral, empresarial, etc.)

\section{Resumiendo lo anterior}

Del conjunto de ideas expuestas hasta aquí en este trabajo, pueden destacarse los puntos siguientes:

(1) El factor fundamental para que una sociedad determinada progrese y se desarrolle, es el Conocimiento y la capacidad efectiva de esa sociedad, para generarlo, difundirlo y aplicarlo, en los diversos campos de la vida social, técnica y científica.

(2) Para lograr lo anterior, el instrumento indispensable es la Educación, pero especialmente, la Universidad y su Investigación; pero no se trata de la universidad en sus formas usuales y tradicionales, sino de todo un repensar de formas nuevas y diferentes, capaces de impulsar tales cambios mediante procesos y teorías de la moderna innovación. 
(3) En la transformación requerida para la nueva universidad, la meta fundamental de ella serían sus aportes progresivos y significativos para impulsar la Sociedad de la Información y del Conocimiento. La Figura 1, "Mapa mental de Nuevas Universidades", proporciona una visión integradora del conjunto de fuerzas y conceptos que deben incidir en una nueva universidad, capaz de propulsar y mantener, el ideal de una sociedad de la información y el conocimiento.

(4) Dada la importancia y complejidad, de la transformación de las universidades y sistemas universitarios actuales, será necesario centrar nuestro examen principalmente, en la triada de los factores de Innovación: Virtualización y Globalización + Formación Personal.

\section{Mapa mental de Nuevas Universidades}

Buzan (2004) define los mapas mentales como "un método de análisis que permite organizar con facilidad los pensamientos y utilizar al máximo las capacidades mentales". En el caso del modelo propuesto de Mapa Mental de nuevas universidades, él intenta ser un modelo de especial utilidad para emprender estudios e investigaciones orientadas hacia la transformación parcial o total de las universidades y conjuntos universitarios actuales. Naturalmente, dentro de cada uno de sus elementos, están implícitamente consideradas las funciones usuales de docencia, investigación y gestión.

El modelo destaca dos ideas; la primera, en lo relativo a considerar fuerzas y conceptos de primordial importancia en cualquier proceso de transformación (parcial o total), algunos de los cuales suelen ser nuevos o poco conocidos en las universidades existentes; ese sería el caso de la Globalización, la Innovación, la Virtualización, la Pertinencia, la Integración y la formación del personal universitario. La segunda idea es que cada uno de los varios conceptos, esté claramente vinculado con lo que se considera ahora que debe ser la meta fundamental de la nueva universidad, es decir el impulso y apoyo continuo al firme desarrollo de la respectiva Sociedad del Conocimiento.

Del conjunto de elementos del mencionado Mapa, analizaremos con mayor detenimiento, sólo cuatro que se consideran los más destacados: Innovación, Virtualización, Globalización y Formación del Personal requerido para la nueva Universidad. Finalmente, también se agregarán algunas aclaraciones acerca de los restantes elementos del Modelo. 


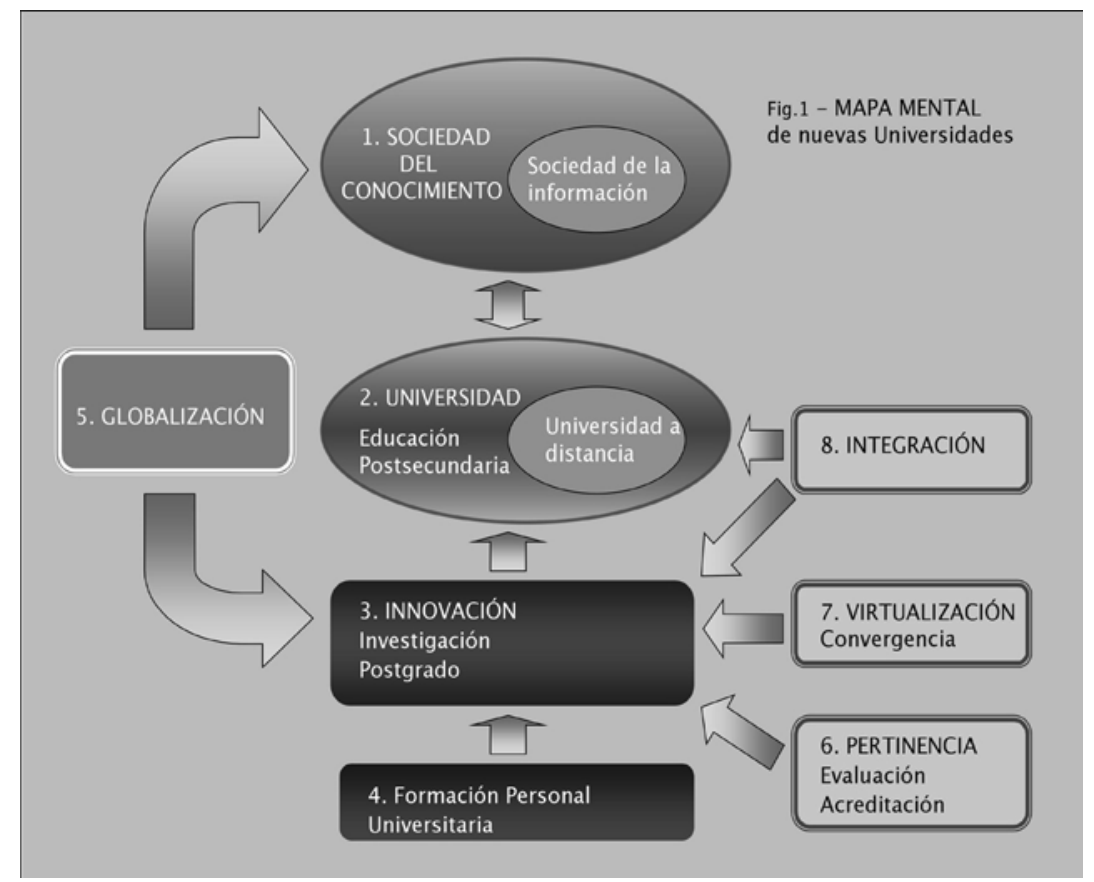

\section{Innovación Universitaria}

"Innovación" no es sinónimo de "Cambio". El Cambio es una mera sustitución de una práctica por otra; por contraste, la Innovación es un "Cambio dirigido con propósitos y claros intentos”, para mejorar, reformar y lograr resultados más efectivos que lo anterior. La Innovación debe ser considerada como fuerza y proceso fundamental para la transformación de la universidad. La virtualización, de las universidades presenciales, a distancia y abiertas, vistas como instrumentos esenciales para impulsar los grandes cambios sociales y científicos permitirá progresar efectivamente hacia la futura Sociedad del Conocimiento. La Innovación, que en el pasado se consideró como un fenómeno individual, esporádico y aislado pasa a ser hoy día un fenómeno social, moderno, colectivo y continuo, en el que la universidad debe asumir un papel clave y conjugar sus actividades científicas y tecnológicas con otras de tipo organizacional, financiero y comercial, para que potencialmente pueda transformar a fondo las estructuras generales y productivas de cualquier sociedad o institución contemporáneas, ya sean desarrolladas o en desarrollo. La Innovación en el Mapa Mental aquí presentado, se encuentra directamente apoyada por la 
Investigación y el Postgrado, pero recibe también la alimentación de: la Globalización, Virtualización, Pertinencia, Integración y Formación del personal de la institución (profesores, investigadores, gestionadores, directivos y técnicos).

Pero pese la extraordinaria importancia de la función de Innovación que debe permear hacia todos los estamentos institucionales, ello no implica ni requiere la creación de megaestructuras rígidas y centralizadas, que desnatulizarían lo esencial de esta función y clima. De lo que se trata es de crear una Cultura de la Innovación que estimule su acción y práctica entusiasta en la mayoría de los sectores, grupos, elementos, niveles y personal de la universidad. Sin esa corriente de formación y opinión favorable de muchos líderes del personal y de los directivos de la universidad, resultaría muy difícil alcanzar una transformación efectiva, que generalmente implica una acción profunda, persistente y con resultados medibles durante el tiempo.

El potencial de aplicación de la Innovación, puede ser usado en diferentes escalas, de redes multinacionales, programas, instituciones, y aún cursos individuales. Entre los ejemplos de mayor tamaño y alcance, podemos nombrar el caso del Estados Unidos, donde como lo cita Hall (1991) en su obra "Access Through Innovation New Colleges for New Students"; él explica que diferentes necesidades de estudiantes fueron tomados muy en cuenta para la creación de los llamados "Land Grant Colleges", en importantes Estados predominantemente agrícolas y rurales. Posteriormente, los importantes avances tecnológicos condujeron a la fundación de University Colleges, con carreras especializadas y generalmente cortas. Asimismo, en las universidades de mayor prestigio apareció el modelo de la Universidad de Investigación, que se estructura en forma muy diferente a las universidades predominantemente docentes. Pero aun dentro de estos diferentes tipos de universidades existe un gran margen para la experimentación, la cual no se practica en sociedades muy centralizadas, generalmente provenientes de regímenes estrictos y rígidos. Existen pocos casos de universidades totalmente virtualizadas, pero en esa categoría podemos señalar a nivel mundial los ejemplos de la Universitat Oberta de Catalunya (España), African Virtual Education (África), National Technological University y la Western Governors University., ambas en los Estados Unidos. Por cierto, que cada una de ellas, con estructuras y procesos de operación sumamente diferentes.

Pero además de estos pocos modelos totalmente virtuales, otro gran número de universidades presenciales, y a distancia tradicional (segunda generación), también están introduciendo cada vez más la virtualización en forma progresiva y en diversos sectores. Este proceso se conoce generalmente con el nombre de "Hibridización", (combinación de lo presencial con lo virtual) y está reseñado en un artículo de Cookson (2002). 


\section{Virtualización}

El extraordinario e incontenible avance de las nuevas tecnologías de Información y Comunicación (NTIC) están modificando profundamente: la organización de las nuevas sociedades y la estructura y orientación del aprendizaje, especialmente el universitario. Entramos a un nuevo mundo, en "La transformación radical: del músculo al cerebro", como lo dicen Arbués \& Tarín (2000), quienes agregan:

"en ese tipo de sociedad, los atributos sociales de generación y procesamiento de la información van más allá del impacto de las tecnologías de información y de la información en si misma. Es la sociedad en su totalidad la que se ve afectada por el nuevo medio técnico“. El aprendizaje es el proceso que nos permite apropiarnos del saber, desarrollarlo y trasnformarlo para aplicarlo de forma individual y colectiva a los problemas y retos que nos plantea la vida económica y social. No saber significa quedar excluido y al margen de la evolución social”.

La sociedad informacional es una organización social en la que la generación, el procesamiento y la transmisión de la información son los factores fundamentales de productividad y poder (Castells, 1996).

Según Silvio (1998), la Virtualización es un proceso y un resultado, al mismo tiempo, del tratamiento de la comunicación mediante ordenadores, de datos, información y conocimiento. Más específicamente, la Virtualización puede comprender la representación de procesos y objetos asociados a actividades de enseñanza y aprendizaje, investigación, extensión y gestión. La Virtualización implica transformar la universidad o partes de ella, para usar funcionalmente el potencial creciente de las TIC sin olvidar que ellas son sólo un medio, pero que el objetivo fundamental es aprender y educar, y que para ello, este medio requiere esencialmente, una racionalidad pedagógica. Como pudimos observar en el punto anterior sobre Innovación Universitaria, la Virtualización representa un elemento importante de Innovación, que puede ser usado en forma integral (universidad totalmente virtual) o para programas o cursos que se desarrollen progresivamente, de acuerdo con un plan estratégico por etapas. En estos casos, el Mapa Mental presenta el contexto a considerar en planes totales o parciales de Innovación y Virtualización Progresivas. 


\section{Convergencia}

En un mundo tan cambiante como el actual se está observando que tanto la Innovación como la Virtualización son cada vez más usados tanto en la universidad presencial, como en las universidades a distancia, abiertas y virtuales. Por consiguiente, puede preverse que la Virtualización acerque y tienda a integrar cada vez más a estas varias modalidades de universidades en una tendencia que se califica ahora como de "Convergencia", que se está desarrollando especialmente en la región asiática, pero que pudiera ser también de mucho interés en países de bajo desarrollo de la Región Iberoamericana. En tal sentido, conocidos expertos como Rumble (1997) y Bates (1999) advierten que no sería improbable que en el futuro tendiera a desaparecer la denominación de universidad a distancia, poniendo así fin a un supuesto litigio entre presencialidad y distancia.

\section{Globalización}

Es una nueva y poderosa fuerza mundial que penetra y modifica la mayoría de los sectores de la sociedad ya sea desarrollada o en desarrollo, y cuya consideración exige sin demoras, transformaciones fundamentales en la educación y especialmente en la universidad. Para vislumbrar los impactos de esta nueva fuerza Castells (1997) expresa que la globalización significa que:

"todos los ámbitos esenciales de nuestra vida están penetrador por actividades cuyo núcleo central se globaliza: la ciencia, la tecnología, los medios de comunicación, los servicios financieros, el arte, el turismo, las profesiones, la música, la cultura, el deporte, la religión, los patrones de consumo e incluso la actividad criminal".

Precisamente en la tesis central de este trabajo sobre la universidad ante la Sociedad del Conocimiento, esta institución superior debe poder responder a los diversos y exigentes planteamientos de la Globalización, mediante respuestas que conduzcan hacia el ideal de la sociedad del conocimiento. Sin embargo, por ahora pocas universidades presentes se están reestructurando decididamente para poder cumplir estos nuevos objetivos universitarios y sociales. Reiterando lo dicho anteriormente, la Innovación, la Virtualización y la Globalización, conjuntamente con la Formación del Personal Universitario, son los factores principales para el vigoroso proceso de transformación de la universidad actual, a fin de que ella pueda cumplir eficazmente con sus nuevas y extraordinarias misiones, tanto sociales como científicas y tecnológicas. 
Miguel CASAS y LiLy STOJANOVIC

INNOVACIÓN Y VIRTUALIZACIÓN PROGRESIVAS DE LAS UNIVERSIDADES IBEROAMERICANAS HACIA LA SOCIEDAD DEL CONOCIMIENTO

\section{Formación del personal universitario}

Si estamos convencidos de la urgente necesidad de profundos cambios en la mayoría de las universidades existentes, el principal obstáculo que se encuentra suele ser la actitud y la preparación de una buena parte de dicho personal universitario (directivos, docentes, investigadores, administradores y técnicos). Para ello, la debida y sólida formación de este personal deberá ser siempre el primer paso en cualquier plan de innovación parcial o total. En ese sentido, Bates (1999) advierte que el problema no consiste tanto en saber cómo usar una tecnología particular, sino en la falta de un marco conceptual adecuado para guiar el uso de la tecnología. De manera simple, esto significa que mucha gente, con la responsabilidad de enseñar, investigar o gestionar, no ha recibido un marco educativo adecuado sobre el que basar su práctica.

\section{Pertinencia}

La UNESCO en varias conferencias Internacionales, ha considerado que la "pertinencia" es un de los factores clave en la educación superior, ya que permite concretar qué cambios esenciales requiere hacer la universidad para que cumpla con su vital contribución a impulsar el saber en la sociedad del conocimiento. La pertinencia da la información necesaria para establecer políticas institucionales, que a medio y largo plazo podrán guiar el avance de la universidad. La "evaluación" y la "acreditación" permitirán constatar, en términos de calidad y cantidad, si las políticas y los planes en ejecución tienen el rumbo necesario.

\section{Integración}

Se parte del supuesto que integrar es mucho más que juntar. La integración requiere que haya un propósito previo y que se formulen unas normas que regulen el funcionamiento del todo y unos indicadores que permitan la posterior y continua evaluación del logro progresivo alcanzado. La integración se aplica a un universo tan amplio como variado, que en las universidades va desde integrar los diversos programas y unidades internas de la institución (p. ej., docencia e investigación), hasta la integración de todo un sistema universitario nacional a la sociedad respectiva o a un área del mundo. Los "conceptos y teorías de sistemas" son un importante apoyo y una útil herramienta para la integración. 


\section{Consideraciones finales}

Estamos conscientes de que pretender visualizar esta región Iberoamericana como un conjunto más o menos homogéneo de países, problemas y posibilidades, no es muy cónsona con la heterogeneidad existente y por ello trataremos de esbozar conceptos generales que en mayor o menor grado tengan alguna vigencia en distintos escenarios e instituciones. El planteamiento central del trabajo es que en diversas sociedades de muy variados niveles de desarrollo y progreso, la universidad debe inevitablemente, hacer un profundo y pronto vuelco en sus objetivos, metas, estructuras y procesos tradicionales, para aprestarse a incidir fundamentalmente en la reorientación de su sociedad, para permitirle alcanzar la condición ideal de moderna sociedad del Conocimiento. Pero este no es un exabrupto tecnológico sino al contrario, como lo dice Castells (2001), "una condición de uso de la tecnología más avanzada para el servicio del bienestar del hombre y de la sociedad, con valores éticos y morales", pero donde la universidad debe contribuir como luz y mente del proceso, ya que la ejecución corresponde a otros estamentos de la sociedad, todos ellos debidamente integrados, como ha sido el extraordinario ejemplo actual de Finlandia, que mediante un Plan Nacional de Innovación, en menos de treinta años, pasó de ser una pequeña sociedad rural y pobre, a convertirse hoy día en un país con los mayores avances tecnológicos, educativos y también de bienestar social, de toda Europa. (Castells \& Imanen, 2002, / Markkula, 1998).

Todas estas extraordinarias transformaciones son ahora posibles a medianos y largos plazos, gracias a los espectaculares avances de la Innovación y de la Virtualización, que facilitan la reestructuración de la universidad convencional y rígida, que lamentablemente en muchos casos, aun sigue dedicándole una parte exagerada de sus recursos y esfuerzos, a la preparación de profesionales de carreras liberales. Sin embargo, la puesta en marcha de estas profundas transformaciones parciales o totales, de la universidad, requerirá de planes estratégicos que impliquen procesos de Innovación y Virtualización en formas progresivas, en vez de improvisadas acciones "efectistas".

\section{Inicio de las transformaciones}

En lo sustantivo, las propuestas de este trabajo tienden hacia la transformación de la universidad actual y por ende también, hacia la transformación de la sociedad para reorientarla cada vez más hacia el ideal de la sociedad de la información y del conocimiento, sea cual sea su nivel de desarrollo actual, pero advirtiendo, que esa 
posible sociedad, aunque será distinta de la actual, en ningún momento ella podrá responder a un modelo rígido, único y universal. Las implicaciones y versatilidad de esa sociedad del conocimiento, resultan explicitadas en las investigaciones y publicaciones principalmente de Manuel Castells, varias de las cuales están reseñadas en la Bibliografía del presente documento. Pero la viabilidad de tan grandes cambios, especialmente en la universidad, no constituirá un proceso simple, técnico, y fácil, pues la universidad por sus propósitos, estructura y gobierno, ha sido y es, una organización conservadora que busca estabilidad y valora seguridad. Como dice Hall (1991), para grandes cambios fruto de la innovación, la universidad enfrentará inevitablemente el conflicto de expectativas entre "Preservación" e "Innovación". Sin embargo, no estamos en el mundo de hace cincuenta o cien años, y una universidad actual, incapaz de transformarse rápidamente, se convertiría inevitablemente en una institución obsoleta y prescindible.

El inicio de cualquier proceso de innovación, debería comprender: un plan integral de innovación (total o parcial) que cuente con las opiniones y entusiasmo, no solamente del equipo directivo sino también de calificados líderes del personal de base ; y un intensivo programa progresivo de formación, para investigadores, docentes, directivos y técnicos, para capacitarlos en el manejo de las nuevas formas académicas y de gestión.

\section{Alternativas de acción}

La situación de la universidad Iberoamericana hoy día presenta una coyuntura histórica, delicada y hasta crucial. En eventos como el presente, fluyen numerosas ideas y avances extraordinarios, que podrían producir cambios y reestructuraciones importantes, pero existe la impresión de que esos conceptos tan avanzados, pocas veces constituyen después una base firme para la acción en instituciones que como hemos visto, están requiriendo grandes decisiones de acción efectiva y continua. Por ello, es nuestra opinión que el tema 3. LA UNIVERSIDAD EN LA SOCIEDAD DEL CONOCIMIENTO, debería ameritar una discusión a fondo y de muy alto nivel para crear una corriente de gran expectativa y disposición hacia el problema de la transformación en las universidades Iberoamericanas.

Las universidades de esta región deben considerar prontamente la posibilidad de entrar en una etapa de transición que plantee grandes cambios para su conjunto y para sus instituciones individuales, cada una de acuerdo con sus condiciones, necesidades y posibilidades. Algunas experiencias de la Unión Europea pueden ser aprovechadas para el sistema y redes pertinentes. El inicio de estos grandes cambios no puede 
esperar porque gracias a ellos, especialmente muchos países latinoamericanos, pueden lograr cambios positivos hacia sociedades verdaderamente democráticas y del conocimiento. El no actuar rápida y efectivamente en el sistema universitario podría implicar una grave perdida de su prestigio y confianza pública e inclusive abrir el camino para la aparición de nuevas formas sustitutivas de aprendizaje, como lo planteó recientemente Buarque (2005) en su presentación de la "Universidad en una Encrucijada" en el Seminario Internacional "La internacionalización del conocimiento: un desafío para las redes de cooperación regionales".

\section{REFERENCIAS BIBLIOGRÁFICAS}

Albornoz, M. (2002) La Universidad ante la Innovación, Aprender para el Futuro. Madrid: Fundación Santillana.

Bates. A. W. (Tony) (1999). La Tecnología en la Enseñanza Abierta y la Educación a Distancia. México: Trillas

Banco Mundial (2000). Red Global de aprendizaje para el Desarrollo Washington: GDLN.

Bello, M. E. (2003) Educación y Globalización. Los discursos educativos en Iberoamérica. Barcelona: Anthropos

Brünner, J. J. (2003) Educación e Internet. ¿La Próxima Revolución? Santiago de Chile. Fondo de Cultura Económica.

Buarque, C. (2005) La Universidad en una Encrucijada. Seminario Internacional: La internacionalización del conocimiento: un desafío para las redes de cooperación regionales. Buenos Aires.

Buzan, T.(2004) Mapas Mentales. Barcelona. URANO.

Casas A. M. Stojanovic, L. (1999) Distance Education: a Decisive force for
Reestructuring the Latinamerican University. CD.ROM. de la XIX Conferencia Mundial del ICDE. Viena

Casas A. M. (2002). Tendencias Actuales e Innovaciones en la Educación Superior a Distancia. Potencialidades y Restricciones en Latinoamérica. Revista Agenda Académica. Caracas UCV.

Casas A. M. (2005) Nueva Universidad ante la Sociedad del Conocimiento. Revista de Universidad y Sociedad del Conocimiento RUSC. Vol. 2, $\mathrm{N}^{\mathrm{O}} 2$. Disponible en: http://www.uoc.edu/ rusc/2/2/dt/esp/casas.pdf [consulta: 15 marzo 2006]

Castells,.M.. (2001) La era de la información: Vol. 1. La Sociedad Red. Madrid: Alianza Editorial

Castells, M. (2001) La Galaxia Internet. Madrid. Arete.

Castells, M.; Himanen, P. (2002) El Estado del Bienestar y la Sociedad de la Información. El Modelo Finlandés. Madrid: Alianza Editorial

Cebrián, M. (coord.) (2003) Enseñanza Virtual para la Innovación Universitaria. Madrid: Narcea. 
Chacón, F. (1997) Un nuevo paradigma para la educación corporativa a distancia CIED. Centro Internacional de Educación y Desarrollo. Asuntos 1: 2. Caracas.

Commission on Standards for School Mathematics (1989). Curriculum and evaluation standards for schools mathematics Ralston, VA: National Council of Teachers of Mathematics.

Cresalc / UNESCO. (1997) La Educación Superior en el Siglo XXI. Visión de América Latina y el Caribe. Tomos I y II. Caracas.

Davis, A (2001) Athabasca University: Conversion from traditional distance education to online Courses, Programs and Services En International Review of Research in Open and Distance Learning. Disponible en: http//www. irrodol.org/content/volau.html. [consulta 3, marzo, 2002].

Diez H., R. (2002) Aprender para el Futuro. Fundación. Madrid: Santillana

Duart, J.; Sangrá, A. (comp) (200o) Aprender en la Virtualidad. UOC. Barcelona: Gedisa

Escotet, M. A. (1991) Aprender para el futuro. Madrid: Fundación Ciencia, Democracia y Sociedad.

Fundación OVSI (2002) Informe sobre la Sociedad de la Información en Iberoamérica. Alicante: Fundación OVSI.

García A., L. (2001) La educación a distancia. De la teoría a la práctica. Barcelona: Ariel.

Hall, W. J. (1991) Access Through Innovation. New Colleges for New Students. New York: Macmillan.
Harasim, L (1993) Global networks. Massachusetts: MIT Press.

Hawkridge, D. (2003) Models for Open and Distance learning. International Research Foundation for Open Learning. Vancouver: The Commonwealth of Learning.

Lundual, B. (ed) (1992) National System of Innovation. Toward a Theory of Innovation and Interactive Learning. Londres. Pinter Publishers.

Markkula, M. (1998) Passión to Learn. Good Lifelong Learning Practice. Helsinki: International Association for Continuing Engineering Education.

Porter.M.E. (1985) Competitive Advantage: creating and sustaining superior performance. New York Free Press.

Queau. Citado en José Silvio (2000): $L a$ Virtualización de la Universidad: ¿Cómo podemos transformar la Educación Superior con la Tecnología? Caracas. IESALC / UNESCO.

Rubio, M. J. (2003) Centro Virtual para el Desarrollo de Estándares de Calidad para la Educación Superior a Distancia en América Latina y el Caribe. Loja: Universidad Técnica Particular de Loja.

Rumble, G. (1997) The cost and economics of Open and Distance Learning. Londres: Kogan Page.

Saragossi, l. (2002) El espacio de investigación Europea y el Nuevo Programa Marco. En: AAVV $L a$ Universidad ante la Innovación. Aprender para el futuro. Madrid: Fundación Santillana. 
Miguel Casas y Lily Stojanovic

INNOVACIÓN Y VIRTUALIZACIÓN PROGRESIVAS DE LAS UNIVERSIDADES IBEROAMERICANAS HACIA LA SOCIEDAD DEL CONOCIMIENTO

Silvio, J. (2000) La virtualización de la Universidad. ¿Cómo podemos transformar la educación superior con la tecnología? Caracas: IESALCUNESCO.

Stojanovic, L. (2003) Las Tecnologías de Información y Comunicación en la Promoción de Nuevas Formas Interactivas $y$ de Aprendizaje en la Educación a Distancia. Agenda Académica. Caracas UCV.

Tünnermann, B., C. (2000) Universidad y Sociedad: Balance histórico $y$ perspectivas desde Latinoamérica. Caracas: UCV.

Tünnermann, C.; De Souza, M. (2003) Desafíos de la Universidad en la Sociedad, del Conocimiento. Cinco años después de la Conferencia Mundial sobre Educación Superior. Paris

Universitat Oberta de Catalunya. (2003) La universidad en la Sociedad del Conocimiento. Barcelona.

Universitad Oberta de Catalunya. (2004) Liderar la Universidad en la Sociedad del Conocimiento E-Learning Objetivo o Estrategia en la gestión del cambio. Barcelona.

Venugopal, R. \& Manjulika, S. (eds) (2002) Toward Virtualization. Open and Distance Learning. New Delhi Kogan Page.

World Bank (1998). World Development Report 1998 / 1999. Knowledge fo Development. Washington

\section{PALABRAS CLAVE}

Innovación, Virtualización, Universidades Latinoamericanas, Sociedad del conocimiento.

\section{KEYWORDS}

Innovation, virtualization, Latinamerican universities, knowledge society.

\section{PERFIL ACADÉMICO DE LOS AUTORES}

Miguel Casas Armengol (Ph. D) Profesor Titular de la Universidad del Zulia (LUZ). Y Rector Fundador de la Universidad Nacional Abierta (UNA). Arquitecto de la Universidad Nacional de Colombia y la Universidad Central de Venezuela. Doctor (Ph. D) en Educación de Stanford University. California. US. "Doctor Honoris Causa" de la Universidad del Zulia. Miembro Activo del Programa de Promoción de Investigadores, de Venezuela. Profesor Invitado de la Stanford University y del Instituto Internacional de Planificación de la UNESCO. Autor de "Universidad sin Clases" Educación a Distancia en América Latina, y capítulos en cinco libros Internacionales y 47 publicaciones en Revistas especializadas arbitradas. armengol 6@hotmail.com telef. (34 93) 2664722 
Miguel CASAS y LiLy STOJANOVIC

INNOVACIÓN Y VIRTUALIZACIÓN PROGRESIVAS DE LAS UNIVERSIDADES IBEROAMERICANAS HACIA LA SOCIEDAD DEL CONOCIMIENTO

Lily Stojanovic (M. A) profesora Titular de la Escuela de Educación UCV y Universidad Nacional Abierta (UNA). Maestría en Educación de la Universidad de Stanford Cal. US. Dos libros sobre evaluación del rendimiento estudiantil y evaluación formativa de materiales instruccionales para la educación a distancia. Su área investigativa está referida al uso de las TIC en educación. Fue Secretaria General de la UNA entre Octubre/1992 a Mayo/1997 2003. Miembro del PPI (Programa de Promoción al Investigador). (Nivel III) FONACIT

Actualmente se desempeña como miembro de la Comisión Técnica del "Área de Tecnologías de Información y Comunicación en Educación” en FONACIT. (Fondo Nacional de Ciencia y Tecnología).

stojanovic@yahoo.com telef.:(58 212) 7814051 Fax: (58 212) 7936802.

Fecha de entrega: 17.10.06

Fecha de aceptación: 03.12.06 